\title{
FATORES SOCIODEMOGRÁFICOS E CLÍNICOS ASSOCIADOS À FORÇA DE PREENSÃO MANUAL E VELOCIDADE DA MARCHA EM LONGEVOS
}

\author{
Maria Helena Lenardt ${ }^{1}$, Clóris Regina Blanski Grden², Jacy Aurelia Vieira de Sousa ${ }^{2}$, Susanne Elero Betiolli \\ Péricles Martim Reche ${ }^{4}$, Tânia Maria Lourenço ${ }^{5}$
}

\begin{abstract}
RESUMO: Pesquisa transversal com o objetivo de investigar a associação entre fatores sociodemográficos e clínicos e os componentes força de preensão manual e velocidade da marcha em idosos longevos. A amostra contemplou 243 longevos, de três Unidades Básicas de Saúde de Curitiba, Paraná, entre janeiro de 2013 e setembro de 2015. Identificou-se associação significativa entre força de preensão manual e sexo $(p=0,001)$, idade $(p=0,001)$, estado civil $(p=0,001)$, moradia $(p=0,001)$, quedas no último ano $(p=0,03)$, perda de urina $(p=0,001)$, uso de bengala $(p=0,001)$ e andador $(p=0,001)$. A velocidade da marcha apresentou associação com sexo $(p=0,001)$, idade $(p=0,001)$, estado civil $(p=0,001)$, moradia $(p=0,001)$, quedas no último ano $(p=0,03)$, perda de urina $(p=0,001)$, uso de bengala $(p=0,001)$ e andador $(p=0,001)$. Destaca-se que a avaliação da força de preensão manual e velocidade da marcha auxiliam na identificação de alterações funcionais em longevos e constitui-se como parte fundamental na gestão da síndrome da fragilidade física.
\end{abstract}

DESCRITORES: Idoso fragilizado; Força da mão; Marcha; Enfermagem geriátrica.

\section{SOCIODEMOGRAPHIC AND CLINICAL FACTORS ASSOCIATED WITH HAND GRIP STRENGTH AND GAIT SPEED IN LONG-LIVING ELDERLY}

\begin{abstract}
Cross-sectional research to investigate the association between sociodemographic and clinical factors and the components hand grip strength and gait speed in long-living elderly. The sample included 243 long-living elderly from three primary health care services in Curitiba, Paraná, between January 2013 and September 2015. A significant association was identified between hand grip strength and sex $(p=0.001)$, age $(p=0.001)$, marital status $(p=0.001)$, housing $(p=0.001)$, falls in previous year ( $p=0.03)$, urine loss $(p=0.001)$, use of cane $(p=0.001)$ and walker $(p=0.001)$. The gait speed was associated with sex $(p=0.001)$, age $(p=0.001)$, marital status $(p=0.001)$, housing $(p=0.001)$, falls in previous year $(p=0.03)$, urine loss $(p=0.001)$, use of cane $(p=0.001)$ and walker $(p=0.001)$. It is highlighted that the assessment of hand grip strength and gait speed helps to identify functional changes in long-living elderly and plays a fundamental role in the management of the physical frailty syndrome.
\end{abstract}

DESCRIPTORS: Frail elderly; Hand strength; Gait; Geriatric nursing.

\section{FACTORES SOCIODEMOGRÁFICOS Y CLÍNICOS ASOCIADOS A LA FUERZA DE PRENSIÓN MANUAL Y VELOCIDAD DE MARCHA EN LONGEVOS}

RESUMEN: Investigación transversal con objeto de investigar la asociación entre factores sociodemográficos y clínicos y los componentes fuerza de prensión manual y velocidad de marcha en ancianos longevos. La muestra incluyó a 243 longevos de tres Unidades Básicas de Salud de Curitiba, Paraná, entre enero de 2013 y septiembre de 2015. Fue identificada asociación significativa entre fuerza de prensión manual y sexo $(p=0,001)$, edad $(p=0,001)$, estado civil $(p=0,001)$, morada ( $p=0,001)$, caídas en el último año $(p=0,03)$, pérdida de orina $(p=0,001)$, uso de bastón $(p=0,001)$ y andador $(p=0,001)$. La velocidad de marcha reveló asociación con sexo $(p=0,001)$, edad $(p=0,001)$, estado civil $(p=0,001)$, morada $(p=0,001)$, caídas en el último año ( $p=0,03)$, pérdida de orina $(p=0,001)$, uso de bengala $(p=0,001)$ y andador $(p=0,001)$. Se destaca que la evaluación de la fuerza de prensión manual y velocidad de marcha ayudan en la identificación de alteraciones funcionales en longevos y representa parte fundamental en la gestión de la síndrome de la fragilidad física.

DESCRIPTORES: Anciano frágil; Fuerza de la mano; Marcha; Enfermería geriátrica.

${ }^{1}$ Enfermeira. Doutora em Filosofia da Enfermagem. Docente do Programa de Pós-graduação em Enfermagem da Universidade Federal do Paraná. Curitiba, PR, Brasil.

${ }^{2}$ Enfermeira. Doutora em Enfermagem. Docente da Universidade Estadual de Ponta Grossa. Ponta Grossa, PR, Brasil.

${ }^{3}$ Enfermeira. Doutora em Enfermagem. Docente de Enfermagem da Universidade Federal do Paraná. Curitiba, PR, Brasil.

${ }^{4}$ Farmacêutico. Doutor em Saúde Coletiva. Docente da Universidade Estadual de Ponta Grossa. Ponta Grossa, PR, Brasil.

${ }^{5}$ Enfermeira. Doutoranda em Enfermagem. Universidade Federal do Paraná. Curitiba, PR, Brasil.

Autor Correspondente:

Recebido: 02/02/2017

Clóris Regina Blanski Grden

Finalizado: 06/07/2017

Universidade Estadual de Ponta Grossa

R. Teodoro Sampaio, 888 - 84036-070 - Ponta Grossa, PR, Brasil

E-mail: crkblanski@uepg.br 


\section{- INTRODUÇÃO}

O incremento do contingente de idosos longevos no Brasil e no mundo tem ampliado o percentual de indivíduos com doenças crônicas, declínio da capacidade funcional e cognitiva, bem como dependência ${ }^{(1)}$. Nesse segmento etário identifica-se aqueles com diminuição das reservas físicas e aumento da vulnerabilidade a estressores internos e externos, culminando para o desenvolvimento da síndrome da fragilidade física ${ }^{(2)}$.

Autores a definem como uma síndrome médica com múltiplas causas e fatores relacionados, caracterizada pela diminuição de força e resistência, bem como pela reduzida função fisiológica que aumenta a vulnerabilidade do indivíduo e desenvolve maior dependência e/ou morte ${ }^{(2-3)}$.

O fenótipo da fragilidade física possui cinco marcadores clínicos evidentes: perda de peso, exaustão autorrelatada, lentidão da marcha, diminuição da força de preensão manual e baixo nível de atividade física $^{(3)}$. Considera-se frágil o idoso que possua três ou mais marcadores da síndrome; a condição pré-frágil contempla indivíduos que apresentem um ou dois componentes; a ausência de todas as características define o idoso como não frágil.

Destaca-se entre os marcadores a força de preensão manual (FPM) e a velocidade da marcha (VM) como importantes preditores do nível de fragilidade física em idosos ${ }^{(4)}$. Como indicadores de força muscular geral, a mensuração destes componentes é considerada instrumento eficiente na predição da morbidade e mortalidade ${ }^{(5)}$.

$\mathrm{Na}$ avaliação da fragilidade física, pesquisadores identificaram esses marcadores como os mais frequentes nos longevos espanhóis( ${ }^{(6)}$, nos idosos americanos do Cardiovascular Health Study ${ }^{(3)}$ e nos participantes do projeto Fragilidade em Idosos Brasileiros (FIBRA) ${ }^{(7)}$. A literatura vigente destaca alguns fatores sociodemográficos e clínicos como contributivos de redução da FPM nos idosos, entre eles o sexo feminino ${ }^{(8)}$, a idade avançada ${ }^{(9)}$ e problemas de saúde ${ }^{(10)}$. Relacionados à redução da VM, os estudos apontam a idade avançada ${ }^{(11)}$, doenças cardiovasculares ${ }^{(12)}$ e quedas $^{(13)}$.

A avaliação dos componentes pelos profissionais de saúde, especificamente os da enfermagem, possibilita a identificação de alterações funcionais e musculares nos longevos. Essas, por sua vez, podem comprometer a realização de tarefas manuais, impor limitações funcionais, predispor a quedas e perda da independência funcional, além de interferir negativamente na capacidade de desempenhar diversas funções essenciais para as atividades diárias dessa população.

Nessa perspectiva, o objetivo do presente estudo foi investigar a associação entre fatores sociodemográficos e clínicos e os componentes força de preensão manual e velocidade da marcha em idosos longevos.

\section{MÉTODO}

Estudo transversal, desenvolvido com idosos longevos ( $\geq 80$ anos) residentes na comunidade, nos domicílios da área de abrangência de três Unidades Básicas de Saúde (UBS) pertencentes ao Distrito Sanitário Boa Vista, da cidade de Curitiba, Paraná, Brasil.

A amostra adotada foi do tipo estratificada proporcional, de modo que nenhuma das UBS foi superestimada ou subestimada. Para o cálculo amostral, considerou-se um poder beta de $80 \%$ (1-ß), nível de significância alfa de 5\% e uma diferença mínima significativa de $10 \%$ entre as proporções de longevos com fragilidade. Da população de 503 longevos, acrescentou-se ao tamanho amostral $10 \%$ pelas possibilidades de perdas e recusas, o que resultou em uma amostra final de 243 longevos (Quadro 1). 
Quadro 1 - Longevos cadastrados nas Unidades Básicas de Saúde investigadas. Curitiba, PR, Brasil, 2015

\begin{tabular}{|l|c|c|c|}
\hline Unidades Básicas de Saúde & População (N) & Participação Proporcional (\%) & Amostra (n) \\
\hline Atuba & 137 & 27,16 & 66 \\
\hline Bairro Alto & 130 & 25,93 & 63 \\
\hline Santa Cândida & 236 & 46,91 & 114 \\
\hline Total & 503 & 100 & 243 \\
\hline
\end{tabular}

Os critérios de inclusão foram: a) idade igual ou superior a 80 anos; b) estar cadastrado em uma das Unidades de Saúde de realização da pesquisa; c) obter pontuação superior ao ponto de corte na aplicação da testagem cognitiva do Mini Exame do Estado Mental (MEEM) ${ }^{(14)}$, sendo 13 pontos para analfabetos, 18 para baixa e média escolaridade e 26 pontos para alta escolaridade ${ }^{(15)}$.

Excluíram-se os longevos: fisicamente incapazes de realizar os testes propostos (acamados, cadeirantes, com membros amputados); em tratamento quimioterápico; e que não possuíssem cuidador familiar presente no momento da visita domiciliar.

Para os longevos que não apresentavam condições cognitivas $(n=36)$ de responder às questões da pesquisa, foi convidado a participar o cuidador familiar, que respondeu às questões sociodemográficas e de autorrelato, para o qual foram elencados os seguintes critérios de inclusão: a) ter idade $\geq 18$ anos; b) ser cuidador familiar; c) residir com o idoso há, pelo menos, três meses.

Foram excluídos os longevos fisicamente incapazes de realizar os testes físicos $(n=15)$ e em tratamento quimioterápico $(\mathrm{n}=\mathrm{um})$.

A pesquisa foi realizada no período de janeiro de 2013 a setembro de 2015 por bolsistas de iniciação científica, mestrandos e doutorandos, os quais receberam treinamento prévio. Um estudo piloto com dez longevos foi conduzido para verificação e adequação do instrumento.

Foram aplicados questionário sociodemográfico adaptado do Instituto de Geografia e Estatística ${ }^{(16)} \mathrm{e}$ clínico inspirado nas seções II e III do questionário multidimensional Brazil Old Age Schedule (BOAS), validado para avaliação da população idosa ${ }^{(17-18)}$, bem como os testes de FPM e VM, avaliados por meio de médias obtidas dos participantes.

As variáveis sociodemográficas investigadas foram: sexo, idade (categorizada em longevos octogenários - $\geq 80$ anos a 89 anos e nonagenários $-\geq 90$ anos a 99 anos), estado civil, escolaridade (categorizada de acordo com os pontos de corte do $\mathrm{MEEM}^{(15)}$ ), arranjo de moradia e situação financeira. As variáveis clínicas incluíram: doenças referidas, uso de medicamentos, hospitalizações, histórico de quedas nos últimos 12 meses e perda de urina. O uso de tecnologias assistivas (bengala, andador, muleta, lentes corretivas) foi incluído na caracterização clínica, por entender que as mesmas influenciam a saúde dos idosos, a maneira com que eles vivem, portanto, significativas para o estudo.

O teste de FPM foi realizado na presença simultânea de dois examinadores, com o dinamômetro hidráulico em quilograma/força (Kgf), da marca Jamar, seguindo a recomendação da American Society of Hand Therapists (ASHT) ${ }^{(19)}$. O longevo permaneceu sentado, com os pés tocando o solo; o membro superior em teste foi posicionado com o ombro em adução, articulação do cotovelo fletida a $90^{\circ}$ e antebraço na posição neutra. A empunhadura foi ajustada na mão dominante do longevo, de forma que a segunda falange do segundo, terceiro e quarto dedos tocasse a curva da haste do dispositivo. $\mathrm{O}$ participante realizou três preensões com a mão dominante, intercaladas por um minuto para retorno da força, e observou-se a medida mais alta ${ }^{(20)}$.

Para avaliar a velocidade da marcha, o idoso foi orientado a caminhar seis metros ${ }^{(5)}$, de maneira habitual, em superfície plana, sinalizada por quatro marcas (início, um metro, cinco metros e fim). Para reduzir os efeitos da aceleração e desaceleração, iniciou-se o teste na segunda marca, interrompendo a cronometragem da caminhada na terceira marca. O cronômetro digital mediu o tempo em segundos para o percurso de quatro metros. A velocidade da marcha foi calculada em metros por segundo $(\mathrm{m} / \mathrm{s})$, conforme estudo internacional sobre fragilidade em idosos ${ }^{(3)}$, a fim de realizar comparações. 
Os dados foram tabulados e analisados por meio do software Stata ${ }^{\circledR}$ versão 12 . Inicialmente os dados foram submetidos à análise exploratória e descritos por medidas de frequência, média e desviopadrão (DP). A normalidade dos dados foi verificada pelo teste de Kolmogorov-Smirnov. Os resultados obtidos pelo referido teste atenderam à pressuposição que os dados tinham distribuição normal. Contemplando ainda os pressupostos, foram realizadas análises de resíduos, os resultados revelaram não haver evidência de que a suposição de homocedasticidade foi violada ou que uma transformação da variável resposta ou das explicativas fosse necessária. Posteriormente, verificou-se a associação entre variáveis independentes e dependente, por meio da regressão linear simples com os testes $\mathrm{F}$ de Fisher e T de Student, utilizando-se para avaliação dos resultados o nível de significância de $p \leq 0,05$.

O desenvolvimento do estudo atendeu às normas nacionais e internacionais de ética em pesquisa envolvendo seres humanos, com aprovação sob o registro CEP/SD: 156.413 do Comitê de Ética em Pesquisa em Seres Humanos do Setor de Ciências da Saúde da Universidade Federal do Paraná.

\section{RESULTADOS}

No que se refere às características sociodemográficas dos longevos, visualiza-se na Tabela 1 que do total de participantes predominou o sexo feminino $(n=161 ; 66,3 \%)$, na faixa etária de $\geq 80$ a 89 anos $(\mathrm{n}=220 ; 90,5 \%)$, com idade mínima de 80 anos e a máxima de 99 anos (média=84,4 43,8 ), viúvos $(n=158 ; 65 \%)$, com baixa escolaridade $(n=137 ; 56,4 \%)$ e que residiam com familiares $(n=144 ; 59,3 \%)$. Dos participantes, 44,5\% ( $n=108)$ consideravam sua situação financeira satisfatória.

Tabela 1 - Distribuição das características sociodemográficas dos idosos longevos. Curitiba, PR, Brasil, 2015

\begin{tabular}{llc} 
Variável & Classificação & Total (\%) \\
\hline \multirow{2}{*}{ Sexo } & Feminino & $161(66,3)$ \\
\cline { 2 - 3 } & Masculino & $82(33,7)$ \\
\hline Idade & $\geq 80$ anos a 89 anos & $220(90,5)$ \\
\cline { 2 - 3 } & $\geq 90$ anos a 99 anos & $23(9,5)$ \\
\hline Estado civil & Viúvo & $158(65)$ \\
\cline { 2 - 3 } & Casado & $73(30)$ \\
\cline { 2 - 3 } Escolaridade* & Solteiro & $12(5)$ \\
\cline { 2 - 3 } & Analfabeto & $90(37)$ \\
\cline { 2 - 3 } & Baixa & $137(56,4)$ \\
\cline { 2 - 3 } & Média & $10(4,1)$ \\
\cline { 2 - 3 } & Alta & $6(2,5)$ \\
\hline Arranjo de moradia & Sozinho & $65(26,7)$ \\
\cline { 2 - 3 } & Com familiares & $144(59,3)$ \\
\cline { 2 - 3 } & Com cônjuge & $34(14)$ \\
\hline Situação financeira & Insatisfatória & $88(36,2)$ \\
\cline { 2 - 3 } & Mediana & $108(44,5)$
\end{tabular}

*Escolaridade: baixa (1-4 anos incompletos); média (4-8 incompletos); alta (8 anos ou mais)

Quanto aos resultados do componente FPM, entre as longevas, o menor e maior valor obtido foram, respectivamente, 4 e $38 \mathrm{Kgf}$, com média de 18,2 Kgf. Para os longevos, a menor força de preensão manual foi de $10 \mathrm{Kgf}$ e a maior de $48 \mathrm{Kgf}$, com média de 28,9 Kgf.

Apresentaram maiores médias de FPM os participantes na faixa etária entre $\geq 80$ a 89 anos (22,1 Kgf), casados (26,1 Kgf), com média escolaridade (22,7 Kgf), que moravam com o cônjuge (26,1 Kgf), com 
situação financeira relatada insatisfatória (22,6 Kgf), que referiram não apresentar incontinência urinária (23,3 Kgf) e que não utilizam andador (22,0 Kgf). As variáveis que apresentaram significância estatística para este componente foram: sexo $(p=0,001)$, idade $(p=0,01)$, estado civil $(p=0,001)$, com quem mora $(p=0,001)$, incontinência urinária $(p=0,001)$ e uso de andador $(p=0,001)$ (Tabela 2$)$.

Tabela 2 - Distribuição das características sociodemográficas e clínicas dos idosos longevos de acordo com as médias e desvio padrão dos marcadores FPM e VM. Curitiba, PR, Brasil, 2015 (continua)

\begin{tabular}{|c|c|c|c|c|c|c|}
\hline & & FPM & & VM & & \\
\hline Variáveis & Total & p-valuet & IC95\% & Total & p-valuet & IC95\% \\
\hline Sexo & & 0,001 & {$[-12,31 ;-9,37]$} & & 0,001 & {$[-0,21 ;-0,57]$} \\
\hline Masculino & $28,9( \pm 7,5)$ & & & $0,70( \pm 0,31)$ & & \\
\hline Feminino & $18,2( \pm 5,2)$ & & & $0,56( \pm 0,30)$ & & \\
\hline Idade & & 0,015 & {$[0,81 ; 7,56]$} & & 0,001 & {$[0,10 ; 0,37]$} \\
\hline$\geq 80$ a 89 anos & $22,1( \pm 7,9)$ & & & $0,63( \pm 0,31)$ & & \\
\hline$\geq 90$ a 99 anos & $18( \pm 7,4)$ & & & $0,39( \pm 0,22)$ & & \\
\hline Estado Civil & & 0,001 & {$[-8,40 ;-4,20]$} & & 0,001 & {$[-028 ;-0,12]$} \\
\hline Casado & $26,1( \pm 8,4)$ & 0,111 & {$[-8,20 ; 0,85]$} & $0,75( \pm 0,33)$ & $\mathbf{0 , 0 0 7}$ & {$[-0,43 ;-0,67]$} \\
\hline Solteiro & $22,4( \pm 7,7)$ & & & $0,50( \pm 0,28)$ & & \\
\hline Viúvo & $19,8( \pm 6,8)$ & & & $0,54( \pm 0,28)$ & & \\
\hline Escolaridade & & 0,939 & {$[-6,84 ; 6,32]$} & & 0,287 & {$[-, 0,12 ; 0,39]$} \\
\hline Alta & $21,3( \pm 6,8)$ & 0,791 & {$[-5,63 ; 7,38]$} & $0,47( \pm 0,26)$ & 0,253 & {$[-, 0,11 ; 0,40]$} \\
\hline Analfabeto & $21,1( \pm 7,5)$ & & & $0,61( \pm 0,33)$ & & {$[-, 0,25 ; 0,37]$} \\
\hline Baixa & $22,2( \pm 8,0)$ & & & $0,61( \pm 0,30)$ & & \\
\hline Média & $22,7( \pm 11,0)$ & & & $0,53( \pm 0,30)$ & & \\
\hline Moradia & & 0,001 & {$[-7,68 ;-1,86]$} & & 0,001 & {$[-, 0,31 ;-0,88]$} \\
\hline Cônjuge & $26,1( \pm 8,3)$ & 0,001 & {$[-8,90 ;-2,46]$} & $0,78( \pm 0,31)$ & 0,001 & {$[-, 0,35 ;-0,11]$} \\
\hline Familiares & $21,4( \pm 7,7)$ & & & $0,58( \pm 0,32)$ & & \\
\hline Sozinho & $20,5( \pm 7,3)$ & & & $0,56( \pm 0,25)$ & & \\
\hline Situação financeira & & 0,352 & {$[-4,14 ; 1,48]$} & & 0,841 & {$[-, 0,01 ; 0,12]$} \\
\hline Insatisfatória & $22,6( \pm 8,0)$ & 0,576 & {$[-3,50 ; 1,95]$} & $0,62( \pm 0,33)$ & 0,361 & {$[-, 0,15 ; 0,57]$} \\
\hline Mediana & $21,3( \pm 7,3)$ & & & $0,63( \pm 0,36)$ & & \\
\hline Satisfatória & $21,8( \pm 8,3)$ & & & $0,57( \pm 0,25)$ & & \\
\hline Doenças & & 0,215 & {$[-10,47 ; 2,36]$} & & 0,837 & {$[-, 0,22 ; 0,27]$} \\
\hline Sim & $21,9(7,9)$ & & & $0,60( \pm 0,31)$ & & \\
\hline Não & $17,8( \pm 5,6)$ & & & $0,63( \pm 0,14)$ & & \\
\hline Medicamentos & & 0,154 & {$[-8,65 ; 1,37]$} & & 0,259 & {$[-, 0,31 ; 0,83]$} \\
\hline Sim & $21,9( \pm 8,0)$ & & & $0,61( \pm 0,31)$ & & \\
\hline Não & $18,3( \pm 4,9)$ & & & $0,50( \pm 0,28)$ & & \\
\hline Quedas (último ano) & & 0,194 & {$[-0,67 ; 3,32]$} & & 0,039 & {$[0,04 ; 0,16]$} \\
\hline Sim & $21,1( \pm 6,6)$ & & & $0,56( \pm 0,32)$ & & \\
\hline Não & $22,4( \pm 8,8)$ & & & $0,64( \pm 0,30)$ & & \\
\hline Hospitalização (último ano) & & 0,097 & {$[-0,38 ; 4,53]$} & & 0,105 & {$[-0,17 ; 0,18]$} \\
\hline Sim & $20,1( \pm 8,3)$ & & & $0,54( \pm 0,25)$ & & \\
\hline Não & $22,2( \pm 7,7)$ & & & $0,62( \pm 0,32)$ & & \\
\hline Perda de urina & & 0,004 & {$[0,91 ; 4,48]$} & & 0,001 & {$[0,76 ; 0,23]$} \\
\hline Sim & $20,4( \pm 6,7)$ & & & $0,53( \pm 0,26)$ & & \\
\hline Não & $23,3( \pm 8,7)$ & & & $0,68( \pm 0,34)$ & & \\
\hline
\end{tabular}




\begin{tabular}{|c|c|c|c|c|c|c|}
\hline Bengala & & 0,092 & {$[-0,37 ; 4,80]$} & & 0,001 & {$[0,10 ; 0,30]$} \\
\hline Sim & $20,0( \pm 7,6)$ & & & $0,44( \pm 0,25)$ & & \\
\hline Não & $22,2( \pm 7,9)$ & & & $0,64( \pm 0,31)$ & & \\
\hline Muleta & & 0,616 & {$[-8,25 ; 13,90]$} & & 0,538 & {$[-0,30 ; 0,56]$} \\
\hline Sim & $19,0( \pm 9,9)$ & & & $0,47( \pm 0,48)$ & & \\
\hline Não & $21,8( \pm 7,9)$ & & & $0,61( \pm 0,31)$ & & \\
\hline Andador & & 0,005 & {$[2,41 ; 13,43]$} & & 0,001 & {$[0,29 ; 071]$} \\
\hline Sim & $14,1( \pm 5,6)$ & & & $0,12( \pm 0,51)$ & & \\
\hline Não & $22,0( \pm 7,8)$ & & & $0,62( \pm 0,30)$ & & \\
\hline Lentes corretivas & & 0,115 & {$[-3,67 ; 0,40]$} & & 0,08 & {$[-0,15 ; 0,09]$} \\
\hline Sim & $22,4( \pm 8,3)$ & & & $0,63( \pm 0,32)$ & & \\
\hline Não & $20,8( \pm 7,2)$ & & & $0,56( \pm 0,29)$ & & \\
\hline
\end{tabular}

† Referente ao valor do teste F de Fisher e t de Student

FPM= Força de preensão manual VM= Velocidade da marcha.

Referente ao marcador velocidade da marcha, considera-se que os maiores valores representam melhor desempenho no teste, ou seja, os longevos mais rápidos. Desta forma, as mulheres obtiveram valores mínimo e máximo de 0,04 m/s e 1,83 m/s, respectivamente, com média de 0,56 m/s. Para os homens obteve-se a menor VM de $0,11 \mathrm{~m} / \mathrm{s}$ e a maior de $1,95 \mathrm{~m} / \mathrm{s}$, com média de $0,70 \mathrm{~m} / \mathrm{s}$.

Para a velocidade da marcha, as maiores médias foram identificadas nos longevos com idade entre $\geq 80$ a 89 anos $(0,63 \mathrm{~m} / \mathrm{s})$, casados $(0,75 \mathrm{~m} / \mathrm{s})$, analfabetos $(0,61 \mathrm{~m} / \mathrm{s})$ e com baixa escolaridade $(0,61 \mathrm{~m} / \mathrm{s})$, que moravam com o cônjuge $(0,78 \mathrm{~m} / \mathrm{s})$, com situação financeira mediana $(0,63 \mathrm{~m} / \mathrm{s})$, que não sofreram quedas no último ano $(0,64 \mathrm{~m} / \mathrm{s})$, que não referiram incontinência urinária $(0,68 \mathrm{~m} / \mathrm{s})$ e que não utilizavam bengala $(0,60 \mathrm{~m} / \mathrm{s})$ ou andador $(0,62 \mathrm{~m} / \mathrm{s})$. As variáveis que mostraram significância estatística para este componente foram: sexo $(p=0,001)$, idade $(p=0,001)$, estado civil $(p=0,001)$, com quem mora $(p=0,001)$, quedas no último ano $(p=0,03)$, incontinência urinária $(p=0,001)$, uso de bengala $(p=0,001)$ e andador $(p=0,001)$ (Tabela 2$)$.

\section{- DISCUSSÃO}

Entre os participantes, o valor médio da FPM foi significativamente maior em homens. Resultados semelhantes foram encontrados em estudos $\operatorname{com}$ idosos $^{(9,21)}$ e longevos ${ }^{(10)}$. Tais achados podem ser atribuídos a maior reserva de massa muscular nos indivíduos do sexo masculino, o que pode justificar a maior produção de força no grupo de longevos.

Independente do sexo, os valores médios de FPM identificados encontram-se nos escores de referência para a faixa etária do estudo ${ }^{(19)}$. Destaca-se que valores de força de preensão manual igual ou inferior a 20 Kgf estão relacionados ao risco para dependência futura e piores condições de saúde ${ }^{(19)}$ com desfecho desfavorável para a funcionalidade dos idosos.

As menores médias de FPM foram constatadas nos participantes com idade entre 90 e 99 anos. A associação estatística significativa entre a idade e o marcador força de preensão manual, identificada neste estudo e em outras investigações ${ }^{(8-9)}$, pode ser explicada pela redução do número e tamanho das fibras musculares, em especial as fibras do tipo II (contração rápida), responsáveis pela produção de força muscular ${ }^{(22)}$. Nos longevos, a prevalência de perda de massa e força muscular é alta e pode comprometer indicadores importantes como a velocidade da marcha, força de preensão manual e a energia $^{(23)}$.

Os longevos casados e que moram com o cônjuge apresentaram médias de FPM significativamente maior que os viúvos e solteiros, que moram sozinhos. Em estudo realizado com idosos coreanos, com objetivo de investigar a associação entre os fatores socioeconômicos e redução da FPM, foi identificado que os idosos casados apresentaram melhores médias de força de preensão manual(24). Compreende- 
se que a condição de viuvez pode contribuir para o isolamento social e familiar, e a falta de estímulo do companheiro pode repercutir nas ações de autocuidado.

Nesta pesquisa, os longevos que não referiram perda de urina apresentaram melhores médias de FPM. Caracterizada como uma síndrome geriátrica, estudos apontam associação significativa entre a incontinência urinária e o marcador força de preensão manual ${ }^{(25)}$.

O uso de tecnologias assistivas pode facilitar a realização das atividades cotidianas pelos idosos e contribuir para o equilíbrio e apoio. Quanto ao andador especificamente, os participantes que não faziam o seu uso apresentaram melhores médias de FPM. No entanto, não foram encontrados estudos que explorassem de modo específico as relações entre o uso dessa tecnologia assistiva e menores médias de FPM.

No presente estudo, os resultados identificados pela VM apontaram que os valores de médias das mulheres $(0,56 \mathrm{~m} / \mathrm{s})$ e dos homens $(0,70 \mathrm{~m} / \mathrm{s})$ encontram-se abaixo do valor padrão $(0,8 \mathrm{~m} / \mathrm{s})$ estabelecido em estudos internacionais ${ }^{(5)}$. Uma justificativa para tais resultados refere-se à característica da amostra (idosos de 80 anos ou mais), a qual pode ter contribuído para redução dos valores encontrados. Por outro lado, com o envelhecimento, há redução de parâmetros envolvidos na marcha como comprimento do passo e velocidade ${ }^{(26)}$. Ressalta-se que médias de velocidade superior ao padronizado predizem melhor expectativa de vida, enquanto que velocidade menor que $0,5 \mathrm{~m} / \mathrm{s}$ prediz pior estado de saúde em idosos ${ }^{(5)}$.

No estudo transversal conduzido com 316 idosos cadastrados na Estratégia Saúde da Família, do município de Lafaiete Coutinho, estado da Bahia, os autores apontam que as mulheres apresentam maior limitação funcional em testes de força/resistência de membros inferiores e locomoção ${ }^{(27)}$. Ainda, identificou-se diferença significativa de médias de velocidade da marcha entre as faixas etárias dos participantes. Nos longevos de 80 a 89 anos, a média de VM foi maior (0,63 m/s) em comparação aos nonagenários de 90 a 99 anos $(0,39 \mathrm{~m} / \mathrm{s})$. A literatura aponta que com o avançar da idade os idosos tendem apresentar menor velocidade da marcha ${ }^{(11)}$.

$\mathrm{Na}$ presente investigação, identificou-se associação estatística significativa entre a idade e os marcadores FPM e VM, do mesmo modo que o estudo transversal, conduzido na cidade de Campinas com 689 idosos da comunidade, cujo objetivo foi investigar relações entre a síndrome da fragilidade e medidas de atividade física ${ }^{(11)}$. Os autores identificaram que a idade avançada (80 anos ou mais) contribui significativamente para a redução da força de preensão manual $(p=0,001)$ e da velocidade da marcha $(p=0,001)$.

Os melhores escores nos parâmetros de VM foram obtidos pelos longevos casados e que moram com o cônjuge. Pode-se afirmar que idosos que se sentem responsáveis pela sua sobrevivência e da família, que se preocupam com o cuidado do parceiro, que mantém uma vida social ativa, tendem a desenvolver mais atividades diárias e serem mais ativos. Por outro lado, idosos que residem sozinhos não tem expectativa de suporte para o cuidado $^{(28)}$, situação que pode influenciar a manutenção da saúde e nas condutas adaptativas em situações de estresse.

Houve associação significativa entre a redução da velocidade da marcha e o autorrelato de quedas no último ano $(\mathrm{p}=0,039)$. O decréscimo de massa muscular associado à idade tem sido apontado como fator importante na relação entre VM reduzida e quedas e pode estar relacionado ao maior desequilíbrio e ao medo de cair ${ }^{(26)}$. No entanto, autores relatam que não há clareza se a redução da marcha ocorre para evitar a queda ou como adaptação ao medo de cair, o que resulta na alteração na marcha $^{(26)}$. Com o envelhecimento, as pessoas tendem a adotar estratégias no intuito de melhorar a estabilidade e evitar o desequilíbrio, a exemplo a realização de passos lentos e curtos.

Os resultados do estudo revelaram associação significativa entre perda de urina e o marcador VM, em que os longevos que possuem continência apresentam as melhores médias de velocidade. Um estudo que teve por objetivo analisar os fatores associados à incontinência urinária entre indivíduos com 60 anos ou mais, com critérios de fragilidade, concluiu que possuir incontinência urinária está associada à pré-fragilidade para qualquer componente ${ }^{(29)}$. Tal condição pode ser considerada como limitador da mobilidade entre os idosos, pois o medo de vivenciar uma situação de constrangimento pode induzi-los a diminuir suas atividades de vida diária, bem como as atividades físicas. 
Quanto ao uso de tecnologias assistivas, houve significância estatística para o uso de bengala $(p=0,001)$ e andador $(p=0,001)$. Esse resultado diverge da pesquisa conduzida com idosos de uma capital do Sul do Brasil, cujo objetivo foi investigar a pré-fragilidade e os fatores associados a essa condição, considerando a velocidade da marcha ${ }^{(12)}$. Destaca-se que o uso desses dispositivos pode constituir-se tanto como facilitadores quanto como barreiras à velocidade e à independência funcional na marcha ${ }^{(30)}$.

O desenho transversal foi um fator limitante da avaliação entre as relações de causa e efeito. Ademais, a amostragem é representativa de uma comunidade local, de modo que não permite generalizar os resultados para outros territórios.

\section{CONSIDERAÇÕES FINAIS}

Identificou-se associação significativa entre as variáveis sociodemográficas e clínicas e os componentes FPM e VM nos idosos longevos. Evidencia-se que tal resultado encontra-se relacionado aos valores das médias obtidas para os componentes supracitados, e que podem contribuir efetivamente para o desenvolvimento da síndrome da fragilidade física nesta população.

Os marcadores investigados interferem significativamente na função muscular, considerada elemento-chave para a saúde global dos idosos, com forte impacto na funcionalidade e na independência. $\mathrm{O}$ cuidado de enfermagem gerontológico deve considerar avaliações frequentes e intervenções minuciosas relacionadas à força dos membros inferiores e superiores. Outro cuidado efetivo relaciona-se ao estímulo à prática de exercícios físicos, especialmente para a prevenção da sarcopenia e contribuição ao retardo do declínio funcional em longevos.

Considerando que a prática de atividade física sofre interferência de fatores fisiológicos, sociodemográficos e clínicos, o cuidado gerontológico também deve ser direcionado para a identificação de barreiras que contribuem para a inatividade física e para a adequação de atividades aos longevos.

A avaliação da FPM e VM dos idosos pelos profissionais de saúde subsidiará o desenvolvimento da gestão do cuidado gerontológico para síndrome da fragilidade física, com o objetivo de minimizar os eventos adversos à saúde e proporcionar impacto positivo na qualidade de vida do idoso longevo.

\section{O REFERÊNCIAS}

1. Age UK. Improving later life: understanding the oldest old.[Internet] 2013 [acesso em 09 jan 2016]. Disponível:http://www.ageuk.org.uk/Documents/EN-GB/For-professionals/Research/Improving\%20Later\%20 Life $\% 202 \% 20$ WEB.pdf?dtrk=true $\% 20$.

2. Morley JE, Vellas B, van Kan GA, Anker SD, Bauer JM, Bernabei R, et al. Frailty consensus: a call to action. J Am Med Dir Assoc. [Internet] 2013;14(6) [acesso em 10 mar 2016]. Disponível: http://dx.doi.org/10.1016/j. jamda.2013.03.022.

3. Fried LP, Tangen CM, Walston J, Newman AB, Hirsch C, Gottdiener J, et al. Frailty in older adults: evidence for a phenotype. J Gerontol A Biol Sci Med Sci. [Internet] 2001;56A(3) [acesso em 22 mar 2016]. Disponível: http:// www.sld.cu/galerias/pdf/sitios/gericuba/fenotipo_frailty.pdf.

4. Odonkor CA, Schonberger RB, Dai F, Shelley KH, Silverman DG, Barash PG. New utility for an old tool: can a simple gait speed test predict ambulatory surgical discharge outcomes? Am J Phys Med Rehabil. [Internet] 2013;92(10) [acesso em 22 mar 2016]. Disponível: http://dx.doi.org/10.1097/PHM.0b013e3182a51ac5.

5. Studenski S, Perera S, Patel K, Rosano C, Faulkner K, Inzitari M, et al. Gait speed and survival in older adults. JAMA.[Internet] 2011;305(1)[acesso em 8 fev 2016]. Disponível:http://dx.doi.org/10.1001/jama.2010.1923.

6. Ferrer A, Formiga F, Sanz H, Monserrate E, Verges D, Grupo Octabaix. Successful aging and indicators of frailty in the elderly. Octabaix Study. Aten Primaria. [Internet] 2014;46(9) [acesso em 18 jun 2016]. Disponível:http:// dx.doi.org/10.1016/j.aprim.2014.01.004. 
7. Fattori A, Neri AL, Santimaria MR, Yassuda MS, Siqueira MEC. Indicadores de Fragilidade. In: Neri AL. (Org). Fragilidade e qualidade de vida na velhice. Campinas, SP: Editora Alínea; 2013.

8. Auyeung TW, Lee SWJ, Leung J, Kwok T, Woo J. Age-associated decline of muscle mass, grip strength hand gait speed: A 4-year longitudinal study of 3018 community-dwelling older Chinese. Geriatr Gerontol Int. [Internet] 2014;14(1) [acesso em 18 jun 2016]. Disponível: http://dx.doi.org/10.1111/ggi.12213.

9. Tveter AT, Dagfinrud H, Moseng T, Holm I. Health-related physical fitness measures: reference values and reference equations for use in clinical practice. Arch Phys Med Rehabil. [Internet] 2014;95(7) [acesso em 20 ago 2016]. Disponível:http://dx.doi.org/10.1016/j.apmr.2014.02.016.

10.Lenardt MH, Grden CRB, de Sousa JAV, Reche PM, Betiolli SE, Ribeiro DKMN.Factors associated with loss of handgrip strength in long-lived elderly. Rev. esc. enferm. USP. [Internet] 2014;48(6) [acesso em 20 ago 2016]. Disponível: http://dx.doi.org/10.1590/S0080-623420140000700007.

11. Bez JPO, Neri AL. Velocidade da marcha, força de preensão e saúde percebida em idosos: dados da Rede FIBRA Campinas, São Paulo, Brasil. Ciênc Saúde Coletiva. [Internet] 2014;19(8) [acesso em 20 dez 2016]. Disponível: http://dx.doi.org/10.1590/1413-81232014198.09592013.

12. Lenardt MH, Carneiro NHK, Betiolli SE, Ribeiro DKMN, Wachholz PA. Prevalence of pre-frailty for the component of gait speed in older adults. Rev. Latino-Am. Enfermagem. [Internet] 2013;21(3) [acesso em 2 dez 2016]. Disponível: http://dx.doi.org/10.1590/S0104-11692013000300012.

13. Clemson L, Kendig H, Mackenzie L, Browning C. Predictors of injurious falls and fear of falling differ: an 11year longitudinal study of incident events in older people. J Aging Health. [Internet] 2015;27(2) [acesso em 2 mar 2016]. Disponível: http://dx.doi.org/10.1177/0898264314546716.

14. Folstein MF, Folstein SE, McHugh PR. "Mini-mental state": a practical method for grading the cognitive state of patients for the clinician. J Psychiatr Res. [Internet] 1975;12(3) [acesso em 10 fev 2016]. Disponível: http://dx.doi. org/10.1016/0022-3956(75)90026-6.

15. Bertolucci PHF, Brucki SMD, Campacci SR, Juliano Y. The Mini-Mental State Examination in a general population: impact of educational status. Arq. Neuro-Psiquiatr. [Internet] 1994;52(1) [acesso em 10 fev 2016]. Disponível: http://dx.doi.org/10.1590/S0004-282X1994000100001.

16. Instituto Brasileiro de Geografia e Estatística (IBGE). Censo Demográfico 2000 - Migração e deslocamento. Questionário da Amostra CD 1.02. [Internet] 2000 [acesso em 17 fev 2015]. Disponível: http://biblioteca.ibge.gov. $\mathrm{br} / \mathrm{pt} / \mathrm{biblioteca-catalogo}$ ?view=detalhes\&id=788.

17. Veras RP,Souza CAM, Cardoso RF, Milioli R, da Silva SD. Pesquisando populações idosas - A importância do instrumento e o treinamento de equipe: uma contribuição metodológica. Rev. Saúde Pública. [Internet] 1988;22(6) [acesso em 10 fev 2016]. Disponível: http://dx.doi.org/10.1590/S0034-89101988000600008.

18. Veras RP. País jovem com cabelos brancos: a saúde do idoso no Brasil. Rio de Janeiro: Relume Dumará; 1994.

19. Bohannon RW, Peolsson A, Massy-Westropp N, Desrosiers J, Bear-Lehman J. Reference values for adult grip strength measured with a Jamar dynamometer: a descriptive meta-analysis. Physiotherapy. [Internet] 2006;92(1) [acesso em 10 fev 2016]. Disponível: http://dx.doi.org/10.1016/j.physio.2005.05.003.

20. Hollak N, Soer R, van der Woude LH, Reneman MF. Towards a comprehensive functional capacity evaluation for hand function. Appl Ergon. [Internet] 2014;45(3) [acesso em 8 fev 2016]. Disponível: http://dx.doi.org/10.1016/j. apergo.2013.09.006.

21. Makizako H, Shimada H, Doi T, Tsutsumimoto K, Suzuki T. Impact of physical frailty on disability in communitydwelling older adults: a prospective cohort study. BMJ Open. [Internet] 2015;5(9) [acesso em 5 ago 2016]. Disponível: http://dx.doi.org/10.1136/bmjopen-2015-008462.

22. Demura S, Aoki H, Sugiura H. Gender differences in hand grip power in the elderly. Arch Gerontol Geriatr. [Internet] 2011;53(1) [acesso em 5 ago 2016]. Disponível: http://dx.doi.org/10.1016/j.archger.2010.06.002.

23. Kent-Braun JA, Callahan DM, Fay JL, Foulis SA, Buonaccorsi JP. Muscle weakness, fatigue, and torque variability: effects of age and mobility status. Muscle Nerve. [Internet] 2014;49(2) [acesso em 12 jun 2016]. Disponível:http:// dx.doi.org/10.1002/mus.23903. 
24. Quan S, Jeong JY, Kim DH. The relationship between smoking, socioeconomic status and grip strength among community-dwelling elderly men in Korea: Hallym Aging Study. Epidemiol Health. [Internet] 2013;(35) [acesso em 12 jun 2016]. Disponível:http://dx.doi.org/10.4178/epih/e2013001.

25. Kim H, Yoshida H, Hu X, Saito K, Yoshida Y, Kim M,et al. Association between self-reported urinary incontinence and musculoskeletal conditions in community-dwelling elderly women: a cross-sectional study. Neurourol Urodyn. [Internet] 2015;34(4) [acesso em 12 jun 2016]. Disponível: http://dx.doi.org/10.1002/nau.22567.

26. Moreira MA, Oliveira BS, de Moura KQ, Tapajós DM, Maciel ACC. Can the gait speed identify elderly with fear of falling?. Rev. bras. geriatr. gerontol.[Internet] 2013;16(1) [acesso em 1 jun 2016]. Disponível:http://dx.doi. org/10.1590/S1809-98232013000100008.

27. Pinheiro PA, Passos TDO, Coqueiro RS, Fernandes MH, Barbosa AR.Motor performance of the elderly in northeast Brazil: differences with ageand sex. Rev. esc. enferm.USP. [Internet] 2013;47(1) [acesso em 1 jun 2016]. Disponível: http://dx.doi.org/10.1590/S0080-62342013000100016.

28. de Oliveira DC, Neri AL, D'Elboux MJ. Variables related to the anticipated support for care in communitydwelling older adults. Rev. Latino-Am. Enfermagem. [Internet] 2013;21(3) [acesso em 10 jun 2016]. Disponível:http:// dx.doi.org/10.1590/S0104-11692013000300013.

29.da Silva VA, D'Elboux MJ. Factors associated with urinary incontinence in elderly individuals who meet frailty criteria. Texto Contexto Enferm. [Internet] 2012;21(2) [acesso em 10 jun 2016]. Disponível:http://dx.doi. org/10.1590/S0104-07072012000200011.

30. Vieira RA, Guerra RO, Giacomin KC, Vasconcelos KSS, Andrade ACS, Pereira LSM, et al. Prevalence of frailty and associated factors in community-dwelling elderly in Belo Horizonte, Minas Gerais State, Brazil: data from the FIBRA study. Cad. Saúde Pública. [Internet] 2013;29(8) [acesso em 1 ago 2016]. Disponível:http://dx.doi. org/10.1590/0102-311X00126312. 